\title{
Association of virulence gene expression with colistin-resistance in Acinetobacter baumannii: analysis of genotype, antimicrobial susceptibility, and biofilm formation
}

\author{
Abbas Bahador 1,5,6 Zahra Farshadzadeh', Reza Raoofian 2,3, Masoumeh Mokhtaran', Babak Pourakbari", \\ Maryam Pourhajibagher ${ }^{1,5}$ and Farhad B. Hashemi ${ }^{*}$
}

\begin{abstract}
Background: Acinetobacter baumannii causes difficult-to-treat nosocomial infections, which often lead to morbidity due to the development of antimicrobial drug resistance and expression of virulence genes. Data regarding the association of resistance to colistin, a last treatment option, and the virulence gene expression of A. baumannii is scarce.

Methods: We evaluated the MLVA genotype, antimicrobial resistance, and biofilm formation of 100 A. baumannii isolates from burn patients, and further compared the in vitro and in vivo expression of four virulence genes among five colistin-resistant A. baumannii (Cst-R-AB) isolates. Five Cst-R-AB isolates were tested; one from the present study, and four isolated previously.

Results: Our results showed that reduced expression of recA, along with increased in vivo expression of IpsB, dnaK, and $b / s A$; are associated with colistin resistance among Cst-R-AB isolates. Differences in virulence gene expressions among Cst-R-AB isolates, may in part explain common discrepant in vitro vs. in vivo susceptibility data during treatment of infections caused by Cst-R-AB.

Conclusions: Our findings highlight the intricate relationship between colistin-resistance and virulence among $A$. baumannii isolates, and underscore the importance of examining the interactions between virulence and antimicrobial resistance toward efforts to control the spread of multidrug-resistant A. baumannii (MDR-AB) isolates, and also to reduce disease severity in burn patients with MDR-AB infection.
\end{abstract}

Keywords: Acinetobacter baumannii, Colistin resistance, Virulence gene expression, Murine burn model

\section{Background}

Acinetobacter baumannii is an opportunistic pathogen that can cause formidable infections among patients with burn wounds worldwide [1]. Also, the World Health Organization (WHO) has announced A. baumannii as "Critical" priority pathogenic bacteria that pose the greatest threat to human health. Treatment of $A$. baumannii

\footnotetext{
*Correspondence: farhadb.hashemi@gmail.com; bonakdar@tums.ac.ir ${ }^{1}$ Department of Microbiology, School of Medicine, Tehran University of Medical Sciences, 100 Poursina Ave., 100 Keshavarz Blvd, Tehran 14167-53955, Iran

Full list of author information is available at the end of the article
}

wound infection is often difficult; primarily due to the pathogenic factors, which enable the establishment of persistent infections within burn patients leading to high morbidity and mortality [2-4]. These pathogenic factors mainly include the development of multi-drug resistance, and production of virulence determinants, such as biofilm formation [5-7]. Reports from various parts of the world have indicated a worrisome growing trend of isolating multi-, extensive- and pan-drug resistant (MDR, XDR, and PDR) strains of $A$. baumannii, some of which are even resistant to colistin, a last resort drug [8-13]. Whether the expression of virulence determinants, such as genes involved in biofilm formation, play an important

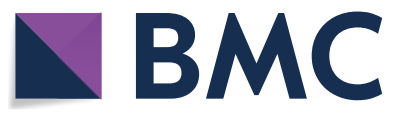

(c) The Author(s) 2018. This article is distributed under the terms of the Creative Commons Attribution 4.0 International License (http://creativecommons.org/licenses/by/4.0/), which permits unrestricted use, distribution, and reproduction in any medium, provided you give appropriate credit to the original author(s) and the source, provide a link to the Creative Commons license, and indicate if changes were made. The Creative Commons Public Domain Dedication waiver (http://creativecommons.org/ publicdomain/zero/1.0/) applies to the data made available in this article, unless otherwise stated. 
role in the high rate of treatment failure among wound infections caused by highly resistant $A$. baumannii isolates remains to be explored [14].

To date, the expression of several $A$. baumannii virulence genes has been linked to the persistence and enhanced survival of $A$. baumannii within the host, including quorum sensing genes [15], and genes that confer biofilm production, which ultimately lead to increased antimicrobial resistance $[16,17]$. Furthermore, the activation of A. baumannii virulence determinant genes, such as rec $A$ and $d n a K$, can render robust strains, which are less vulnerable to host response stresses [18]. Moreover, the expression of recA by $A$. baumannii isolates increases resistance to stress [19], while $r e c A$ inactivation increases susceptibility to a variety of antimicrobial agents $[15,20]$.

Then again, mounting evidence suggests that increased virulence gives rise to isolates that are less "fit" to survive in their host, and renders them more susceptible to antimicrobial agents [21]. For instance, the loss of glycosyl transferase gene (lpsB)], essential for A. baumannii lipopolysaccharide (LPS) core biosynthesis, corresponds to the phenotypes of "reduced survival rate", attenuated biofilm formation, and increased antimicrobial susceptibility [22-24]. Similarly, the expression of blue light sensing $(b l s A)$ gene and production of BlsA protein has been demonstrated to inhibit biofilm formation, which also leads to increased susceptibility to antimicrobial agents $[25,26]$. In addition, among A. baumannii clinical isolates, the development of colistin resistance $\left(\mathrm{Cst}^{\mathrm{R}}\right)$ has been shown to enhance biofilm formation, but reduce their invasiveness $[27,28]$. Although these reports present evidence of likely interactions between virulence and the antimicrobial susceptibility profile of $A$. baumannii, the association of the expression of specific virulence genes with antimicrobial susceptibility has not been thoroughly investigated. Likewise, data comparing the expression of virulence genes among colistin-resistant $A$. baumannii (Cst-R-AB) strains is scarce.

In the present study, we aim to examine whether resistance to colistin in A. baumannii isolates is associated with the expression of specific virulence genes. We also analyze the genotypes of the A. baumannii isolates, their biofilm formation ability, and antimicrobial susceptibility profiles. By revealing the interaction of virulence genes with $\mathrm{Cst}^{\mathrm{R}}$ among $A$. baumannii isolates, we attempt to explain, at least in part, the apparent discrepancies between in vitro antimicrobial resistance data, and in vivo clinical outcomes in A. baumannii infected burn patients. Our findings may ultimately help devise strategies toward effective treatments of $A$. baumannii burn wound infections, as well as make prudent decisions regarding other infection control measures to thwart nosocomial outbreaks of MDR A. baumannii.

\section{Methods}

\section{Specimens, bacterial isolates and cultures}

One hundred non-repetitive clinical specimens were collected from the burn unit of the Burn Medical Center Complex in Tehran, Iran; during April-December 2014. After admission, written informed consent forms were obtained from either the patients, or their authorized representatives. This study was approved by Tehran University of Medical Sciences (TUMS) Research Ethics Committee (Application No. TREC-89. 01-30-10430). All 100 burn wound isolates were initially identified as A. baumannii using the API20NE system (bioMérieux, Marcy-l'Etoile, France), and later confirmed using the gyrB multiplex PCR, as described previously [29]. Mueller-Hinton Broth (MHB) and brain heart infusion (BHI) agar plates (both from Merck, Darmstadt, Germany) were used to culture the bacterial isolates under aerobic conditions for $24 \mathrm{~h}$ at $37{ }^{\circ} \mathrm{C}$. In total, five Cst-R-AB isolates were analyzed including, a single Cst- $R-A B$ isolates from this study, and four additional Cst-R-AB isolates from previous cohort studies.

\section{Antimicrobial susceptibility testing}

To assess antimicrobial susceptibility patterns of $A$. baumannii clinical isolates, we carried out the disk agar diffusion (DAD) method according to the Clinical and Laboratory Standards Institute (CLSI) procedures [30] and breakpoint interpretations, using antimicrobial disks containing the 17 antimicrobial agents: ampicillin-sulbactam, cefepime, ceftazidime, ciprofloxacin, colistin, gentamicin, imipenem, levofloxacin, meropenem, minocycline, piperacillin, piperacillin-tazobactam, rifampicin, tetracycline, tigecycline, tobramycin, and trimethoprimsulfamethoxazole (Mast Diagnostics, Bootle, UK). The CLSI guideline for Broth microdilution test for minimum inhibitory concentration (MIC) was used to assess isolate susceptibility to colistin, rifampicin, and tigecycline. Colistin MICs were interpreted according to the CLSI breakpoints [30]. For tigecycline susceptibility tests, the criteria of the European Committee on Antimicrobial Susceptibility Testing (EUCAST) [31] for Enterobacteriaceae were used, in which an $\mathrm{MIC}$ of $<1 \mu \mathrm{g} / \mathrm{mL}$ was defined as susceptible and $>2 \mu \mathrm{g} / \mathrm{mL}$ was considered resistant [31]. Rifampicin susceptibility was interpreted according to CLSI criteria using breakpoint values suggested for Staphylococcus aureus, in which susceptible and resistant were defined as $\leq 1$ and $\geq 4 \mu \mathrm{g} / \mathrm{mL}$, respectively [30]. A. baumannii isolates were defined as multi-, extensive- and pan-drug resistant (MDR, XDR, and PDR) according to the definitions described previously [32]. Antimicrobial agents were categorized into three groups; namely, Group A that were agents deemed appropriate for primary testing panel; Group B agents, 
for which primary testing may be warranted, but they may be reported selectively (such as when the organism is resistant to agents of the same class, as in group A). Also, Group O (other) that comprised agents that had a clinical indication for A. baumannii, but were not candidates for primary testing panel in the USA [30]. Colistin resistance $\left(\mathrm{Cst}^{\mathrm{R}}\right)$ among $A$. baumannii isolates was confirmed by the genetic analysis of the $p m r$ operon showing mutations in $p m r A / p m r B$ signal transduction system, which confers $\mathrm{Cst}^{\mathrm{R}}$ by modifying lipid A phosphoethanolamine moiety of the lipopolysaccharide (LPS) layer in the outer membrane.

\section{International clones, MLVA genotype analysis}

International clone (IC) types of the isolates were determined based on the presence of ompA, csuE, and bla $a_{\mathrm{OXA}}$ ${ }_{51}$-like allele amplicons using two complementary multiplex PCR assays, as previously described [33]. Table 1 shows the primers used in the multiplex PCR assays, which selectively amplified the outer membrane protein A (ompA), chaperone-subunit usher $\mathrm{E}$ (csuE), and $b l a_{\text {OXA-51- }}$ like intrinsic carbapenemase genes of the A. baumannii isolates. Isolates not assigned as either IC type I, II, or type III were reported as the variant clonal type (IC-V).

To examine the genotypic diversity of A. baumannii clinical isolates, multi-loci variable-number tandem repeat analysis (MLVA) was carried out, as previously described [34]. Briefly, DNA from the A. baumannii isolates was extracted using a GeneJET DNA purification kit and its concentration was assessed using a NanoDrop 1000 spectrophotometer (Thermo Scientific, USA). Genomic DNA $(25 \mathrm{ng} / \mu \mathrm{L})$ was assayed by PCR using a $30 \mu \mathrm{L}$ final volume containing $5 \mathrm{ng}$ of DNA, $10 \times$ reaction buffer (SinaClon BioScience, Iran), $1.5 \mathrm{mM} \mathrm{MgCl}$ $[1,2] \mathrm{U}$ of Taq DNA polymerase, $200 \mu \mathrm{M}$ each deoxynucleotides (dNTP), and $0.3 \mu \mathrm{M}$ of each primer (all provided by SinaClon BioScience, Iran). Amplification cycles included an initial $94{ }^{\circ} \mathrm{C}$ denaturation for $5 \mathrm{~min}$,

Table 1 Primer sequences used in this study

\begin{tabular}{lll}
\hline Primer name & Sequence $\left(\mathbf{5}^{\prime} \mathbf{-} \mathbf{3}^{\prime} \mathbf{)}\right.$ & $\begin{array}{l}\text { Amplicon } \\
\text { size } \mathbf{( b p )}\end{array}$ \\
\hline $\begin{array}{l}\text { blsA-F } \\
\text { blsA-R }\end{array}$ & ACCTTTAACCCGCTTTTGCT & 117 \\
IpsB-F & TCCCCTATTCACCATTCCAA & \\
IpsB-R & AGGCCATCAATCTTTGGTTG & 137 \\
dnak-F & GCTGACGTAATGGACGGATT & \\
dnak-R & GCGTTTAATTGGTCGTCGTT & 105 \\
recA-F & ACTTCAACCCAAGCATCACC & \\
recA-R & CACGCCCTAGACCCTCAATA & 136 \\
$16 s r R N A-F$ & CGATTAAATCAATTGCGCCT & \\
$16 s r R N A-R$ & AAAGTTGGTATTCGCAACGG & 117 \\
\hline
\end{tabular}

followed by 35 cycles of $94{ }^{\circ} \mathrm{C}$ for $30,30 \mathrm{~s}$ of annealing at 50 or $55^{\circ} \mathrm{C}$ depend on melting temperature of the primers, elongation at $72{ }^{\circ} \mathrm{C}$ for $30 \mathrm{~s}$, and a final elongation at $72{ }^{\circ} \mathrm{C}$ for $10 \mathrm{~min}$. Then, $5 \mu \mathrm{L}$ of each PCR amplicon solution were analyzed on a $25 \mathrm{~cm} 3 \%$ agarose gel (SinaClon BioScience, Iran) by electrophoresis $(3 \mathrm{~h}$ at $6 \mathrm{~V} / \mathrm{cm})$ in $1 \times$ Tris-borate-EDTA (TBE) buffer. Size markers included a 100-bp (SinaClon Bioscience), and 50-bp DNA standards (Thermo Scientific) for L- and S-variable number tandem repeats (VNTRs), respectively. The amplicon bands were visualized with UV illumination after ethidium bromide $(0.5 \mu \mathrm{g} / \mathrm{mL})$ staining. Band sizes were determined using GeneTools v.3.08 automatic image analysis software (Syngene, Cambridge, United Kingdom). For cluster analysis, allele strings were analyzed by BioNumerics software v.7.0 as character values (Applied Maths, Sint-Martens-Latem, Belgium). Clusters were defined using cut-off values of 95\% similarity, and MLVA type was determined using a $100 \%$ similarity cutoff, as previously described [35].

\section{Semi-quantitative biofilm formation assay}

The semi-quantitative assessment of biofilm formation was performed in triplicate using crystal violet staining, as previously described [36]. Briefly, A. baumannii isolates were cultured on BHI agar overnight, and an isolated colony was suspended in LB Broth (Himedia, India) for $4 \mathrm{~h}$. Bacterial suspensions $(200 \mu \mathrm{L})$ in logarithmic growth phase were adjusted to 0.5 McFarland's standard $\left[1.5 \times 10^{8}\right.$ colony forming units $\left.(\mathrm{CFU}) / \mathrm{mL}\right]$ and incubated at $35{ }^{\circ} \mathrm{C}$ in flat-bottomed 96-well plates. After $48 \mathrm{~h}$, media was removed, and wells were washed three times with $\mathrm{dH}_{2} \mathrm{O}$. Then, $200 \mu \mathrm{L}$ of $1 \%$ crystal violet dye was added and plates were incubated at room temperature (RT) for $20 \mathrm{~min}$. The dye was then decanted, wells were washed twice with $\mathrm{dH}_{2} \mathrm{O}$ and dried. Finally, $200 \mu \mathrm{L}$ of $95 \%$ ethanol $(200 \mu \mathrm{L} /$ well) was added, and the optical absorbance (A) was measured at $570 \mathrm{~nm}$ (Thermo Scientific GmbH, Driesch, Germany). Reference strain (ATCC $\mathrm{DH} 5 \alpha)$ was used as negative control (NC) to assign scores for biofilm formation, according to following formula: negative isolate $(N)=A_{I} \leq A_{N C}$; weakly positive isolate $(W)=A_{N C}<A_{I} \leq 2 A_{N C}$; moderately positive isolate $(\mathrm{M})=2 \mathrm{~A}_{\mathrm{NC}}<\mathrm{A}_{\mathrm{I}} \leq 4 \mathrm{~A}_{\mathrm{NC}}$; and strongly positive isolate $(\mathrm{S})=\mathrm{A}_{\mathrm{I}}>4 \mathrm{~A}_{\mathrm{NC}}$.

\section{Virulence genes detection}

To detect virulence factor genes $d n a K, \operatorname{rec} A, l p s B$ and $b l s A$, a series of PCR assays were utilized using primers (Table 1; Primer 3 software v.4.0). Briefly, $1 \mu \mathrm{L}$ of genomic DNA ( $25 \mathrm{ng}$ ) from Cst-R-AB strains was assessed by PCR using $12.5 \mu \mathrm{L}$ of $2 \times$ PCR Master Mix, and $1 \mu \mathrm{L}(2.5 \mathrm{pM})$ of each primer (both from SinaClon BioScience, Iran). Amplifications were performed using a Mastercycler 
Personal (Eppendorf ${ }^{\circledR}$, Germany), with initial denaturation for $5 \mathrm{~min}$ at $94{ }^{\circ} \mathrm{C}$, and 35 cycles at $94{ }^{\circ} \mathrm{C}$ for $30 \mathrm{~s}$, annealing for $30 \mathrm{~s}$ at $52{ }^{\circ} \mathrm{C}$ (Table 1), followed by elongation at $72{ }^{\circ} \mathrm{C}$ for $30 \mathrm{~s}$, and a final step at $72{ }^{\circ} \mathrm{C}$ for $5 \mathrm{~min}$. The presence of gene specific amplicons was verified visually by $2 \%$ agarose gel electrophoresis using ethidium bromide staining.

\section{Animal study design}

All animal experiments were carried out in accordance to the protocols approved by the Animal Ethics Committee of Tehran University of Medical Sciences (Application No. TUMS-AEC-89-0130-10430). Male C57BL/6 mice (6-8 week old; $18-23$ g; Pasteur Institute, Tehran, Iran) were housed one mouse per cage, under sanitary conditions at $22-25{ }^{\circ} \mathrm{C}$ and at $12 \mathrm{~h}$ light/dark cycles, with access to sanitized pellet food and water. Mice were acclimated to room conditions for 1 week prior to each experiment. To increase the accuracy of microbiological assessments, the cages were disinfected with povidone iodine solution $10 \%$, and the bedding materials were autoclaved, and replaced every day.

Based on mortality rates of burn wound infection from previous studies, the effect size (No. of mice in experimental groups) was estimated as five mice per each isolate, using power analysis with power arbitrarily set at $80 \%$. Initially, two groups (five mice per group) were infected with the only Cst-R-AB (MT10) isolated in this study. One group was infected with colistin-treated Cst$\mathrm{R}-\mathrm{AB}$ isolate, and the other with Cst- $\mathrm{R}-\mathrm{AB}$ isolate with no colistin treatment. Mock-infected burned mice (C-Burn) served as controls, and received physiological saline instead of $A$. baumannii infection (third group).

Similarly, groups of mice were later infected with additional four Cst-R-AB isolates (MIC 32-256 $\mu \mathrm{g} / \mathrm{mL}$ ), recovered from burn wounds in a previous study [37]. In order to expose the Cst-R-AB isolates to colistin, and evaluate the putative colistin-induced changes in virulence gene expression, the group of colistin-treated Cst$\mathrm{R}-\mathrm{AB}$ infected mice were administered with colistin (20 $\mu \mathrm{g} / \mathrm{g}$ weight); which yielded sub-MIC serum levels of approximately $25 \mu \mathrm{g} / \mu \mathrm{L}$ [38]. For analysis of all five (i.e. $1+4)$ Cst-R-AB isolates, all in vivo data were combined and presented as Mean $+\mathrm{SD}$.

\section{Murine burn wound infection and bacterial quantification}

Burn wounds were generated, as described previously [39]. Briefly, mice were anesthetized by an intraperitoneal (i.p.) injection of a ketamine-xylazine cocktail, after which the dorsal/posterior surface was shaved, and each mouse was then injected with $0.5 \mathrm{~mL}$ (i.p.) of sterile saline to prevent dehydration. A brass $10 \mathrm{~mm} \times 10 \mathrm{~mm}$ block preheated to $95{ }^{\circ} \mathrm{C}$ was applied to the shaved area for a $10 \mathrm{~s}$ period. This procedure consistently generated a nonlethal, full-thickness, third-degree burn. Burn wound infections were established as previously described [40]. Briefly, 5 min after inducing the burn wound, using a sterile inoculating loop, a $50 \mu \mathrm{L}$ suspension of each Cst$\mathrm{R}-\mathrm{AB}$ isolate containing $1.1-2.1 \times 10^{5} \mathrm{CFU} / \mathrm{mL}$ was smeared onto the burn wound site. After $72 \mathrm{~h}$, bacterial concentrations in wound sites of mice infected with untreated Cst-R-AB were compared to mice infected with Cst-treated Cst-R-AB isolate (treated with sub-optimal i.e. <MIC). Viable bacteria quantitated by colony enumeration of bacteria harvests from the wound bed, and reported as $\mathrm{CFU} / \mathrm{mL}$ by using biopsy punch method as previously described [41]. All mice were euthanized via an overdose of ketamine $(250 \mathrm{mg} / \mathrm{kg})$ and xylazine $(25 \mathrm{mg} / \mathrm{kg}$ ) prior to bacterial harvest from the wound site.

\section{RNA extraction and CDNA synthesis and in vivo virulence gene expression}

To measure in vivo virulence genes expression within the wound bed skin, tissue specimens were collected from the infected tissue from each sacrificed mouse were processed separately. Briefly, wound bed skin tissue specimens were aseptically excised ( $2 \mathrm{~mm}$ deep), placed in PBS, and frozen in liquid $\mathrm{N}_{2}$ for immediate RNA extraction.

To compare the relative quantities of gene-specific mRNA of Cst-R-AB isolates under in vivo vs. in vitro conditions RNA extraction was carried out. For the "in vivo samples" mRNA was extracted from Cst-R-AB isolates in their respective burn wound sites, $72 \mathrm{~h}$ after inoculation. The in vitro mRNA samples were, however, prepared using Cst-R-AB isolates grown in culture media, as described above. In all comparative analyses, 16S rRNA gene mRNA served as control to normalize mRNA quantities among the samples. Total bacterial RNA of planktonic mid-log phase cultures of Cst-R-AB isolates were extracted using the Total RNA Extraction Kit (iNtRON Biotech Inc., Seoul, South Korea). To extract bacterial RNA from tissue, wound specimens were ground and homogenized in liquid nitrogen, and the cellular lysates were run through All Prep DNA/RNA columns. Total RNA extraction was performed using RNeasy Plus Mini Kit (QIAGEN, Hilden, Germany) following the manufacturer's recommendations. Bacterial mRNA enrichment was achieved by step-wise treatment of total RNA samples to eliminate mouse mRNA [NEBNext Poly(A) mRNA Magnetic Isolation Module, New England Biolabs, USA], followed by the removal of mouse and bacterial rRNA (Ribo-Zero Gold rRNA Removal Kit, Epicentre Biotechnologies, USA). RNA purity was determined by $260 / 280$ and $260 / 230 \mathrm{~nm}$ absorbance ratio using a NanoDrop 1000 spectrophotometer (Thermo Scientific, USA), and size-verified by $2 \%$ agarose gel electrophoresis 
prior to RT-PCR and qRT-PCR assays. Before generating cDNA, bacterial mRNA samples were treated with DNase I (Thermo Scientific, USA) and RevertAid First Strand cDNA Synthesis Kit (Thermo Scientific, USA). Samples of $1 \mu \mathrm{g}$ total RNA were used to generate cDNA libraries, for the reverse transcription (RT)-PCR, and quantitative real time RT-PCR (qRT-PCR) assays.

\section{Reverse transcriptase (RT-PCR) and quantitative (qRT)-PCR assays}

RT-PCR assay was used to assess the expression of $d n a K$, $b l s A$, rec $A$, and $l p s B$ genes. Briefly, first-strand cDNA served as a template for the amplification of virulence genes, and qRT-PCR was used to measure the relative changes in mRNA levels as indicators of virulence gene expression. Samples of cDNA from Cst-R-AB isolates were analyzed by qRT-PCR assay using the primer sets shown in Table 1 . The Line-Gene K Real Time PCR System (BIOER Technology Co., China) was used to perform the qRT-PCR according to the minimum information for publication of quantitative real-time PCR experiments (MIQE) guidelines [42] under the following conditions: $95^{\circ} \mathrm{C}$ for $15 \mathrm{~min}$, followed by 45 cycles of $95^{\circ} \mathrm{C}$ for $15 \mathrm{~s}$, annealing for $30 \mathrm{~s}$ at 60 , and $72{ }^{\circ} \mathrm{C}$ for 30 s, using $10 \mu \mathrm{L}$ of SYBR $^{\circledR}$ Premix Ex Taq ${ }^{\mathrm{TM}}$ II (Tli RNaseH Plus) (TaKaRa Bio Inc., Japan), $2 \mu \mathrm{L}$ of gene-specific forward and reverse primers (2.5 pmol), cDNA template (50 ng), and $7 \mu \mathrm{L}$ of $\mathrm{ddH}_{2} \mathrm{O}$ (total $20 \mu \mathrm{L}$ ). The quantity of gene-specific amplicons was determined by qPCR relative to an internal standard (16S rRNA) used as a calibrator. Target amplicons were checked for size and specificity by agarose gel electrophoresis and melting curve analysis, respectively. The changes in the expression level of each virulence gene was calculated by the $2^{-\Delta \Delta \mathrm{Ct}}$ method using the Relative Expression Software Tool (REST) 2009 software (version 2.0.13; Qiagen, Valencia, CA, USA) [44]. Difference of $>4$-fold relative to basal gene expression levels was considered significant, and referred to as $n$-fold difference.

\section{Statistical analysis}

Data were analyzed using the Student's t-test, and the Chi square test using the SPSS software package (version 22). Results were considered significant if $P<0.05$.

\section{Results}

\section{Antimicrobial susceptibility profiles}

As shown in Fig. 1, the majority (95\%) of A. baumannii isolates had a MDR (31\%) or XDR (64\%) profile of antimicrobial resistance, while the frequency of non-MDR, or PDR isolates was $4 \%$, and $1 \%$, respectively. While most isolates (96\%) were resistant to at least three classes of antimicrobial agents, nearly $90 \%$ were resistant to at least 10 tested antimicrobial agents including; cephalosporins, trimetho$\mathrm{prim} / \mathrm{sulfamethoxazole,} \mathrm{tetracycline,} \mathrm{piperacillin/tazo-}$ bactam, and fluoroquinolones. Figure 1 also shows the susceptibility profile of $A$. baumannii isolates against seven antimicrobial agents representing the $\mathrm{A}, \mathrm{B}$, and $\mathrm{O}$ groups of antimicrobial agents [30]. The highest resistance rates were among group A (45-96\%), followed by group B (19-97\%), and group $\mathrm{O}$ antimicrobial agents (1\%). The lowest rates of resistance were against colistin (1\%), tigecycline (19\%), and minocycline (42\%). Moreover, resistance rates to ciprofloxacin, tobramycin, and imipenem were 89,64 and, $60 \%$ respectively. Surprisingly, the rate of resistance to tobramycin was higher among MDR isolates (82\%) than the XDR $(87 \%)$ isolates $(P>0.05)$.

\section{MLVA genotyping and international clone analysis}

International clone (IC) type analysis showed that the majority of $A$. baumannii isolates were IC-V variants (43\%), followed by IC-II (37\%), IC-I (19\%), and IC-III types (1\%). Also, MLVA genotype analysis ( $95 \%$ allelic similarity) revealed eight different strain clusters, including strain clusters A, B, and C in one group, as well as clusters D through $\mathrm{H}$ in another group (Fig. 1).

Overall, we identified 32 MLVA types (MT), which consisted of 1-24 members each (at 100\% similarity cutoff). While most MTs had a single member, the three main MTs were MT29 $(n=24)$, MT13 $(n=17)$, and MT14 $(n=10)$, which together comprised over $50 \%$ of all isolates (Fig. 1). The majority of MT29 (18/24) and MT14 (8/10) members were IC-V variants, which were predominantly XDR isolates. Specifically, 83\% (15/18) of MT29 IC-V variants, and 50\% (4/8) of MT14 variants showed XDR profiles. The small MT12 cluster $(\mathrm{n}=4)$ was remarkable since all four were IC-V and XDR isolates. Approximately $75 \%$ of the MT29 isolates and 40\% of the MT14 isolates were categorized as IC-V. Among the MT29, MT13, and MT14 isolates, XDR isolates predominated and showed similar frequencies. Most MT13 isolates $(76 \%)$ were IC-II types with nearly $70 \%$ of these being XDR strains (Fig. 1).

\section{Association of MDR profile with IC types and biofilm formation index}

We further examined the association of the MDR profile of antimicrobial resistance of each isolate with the ability 


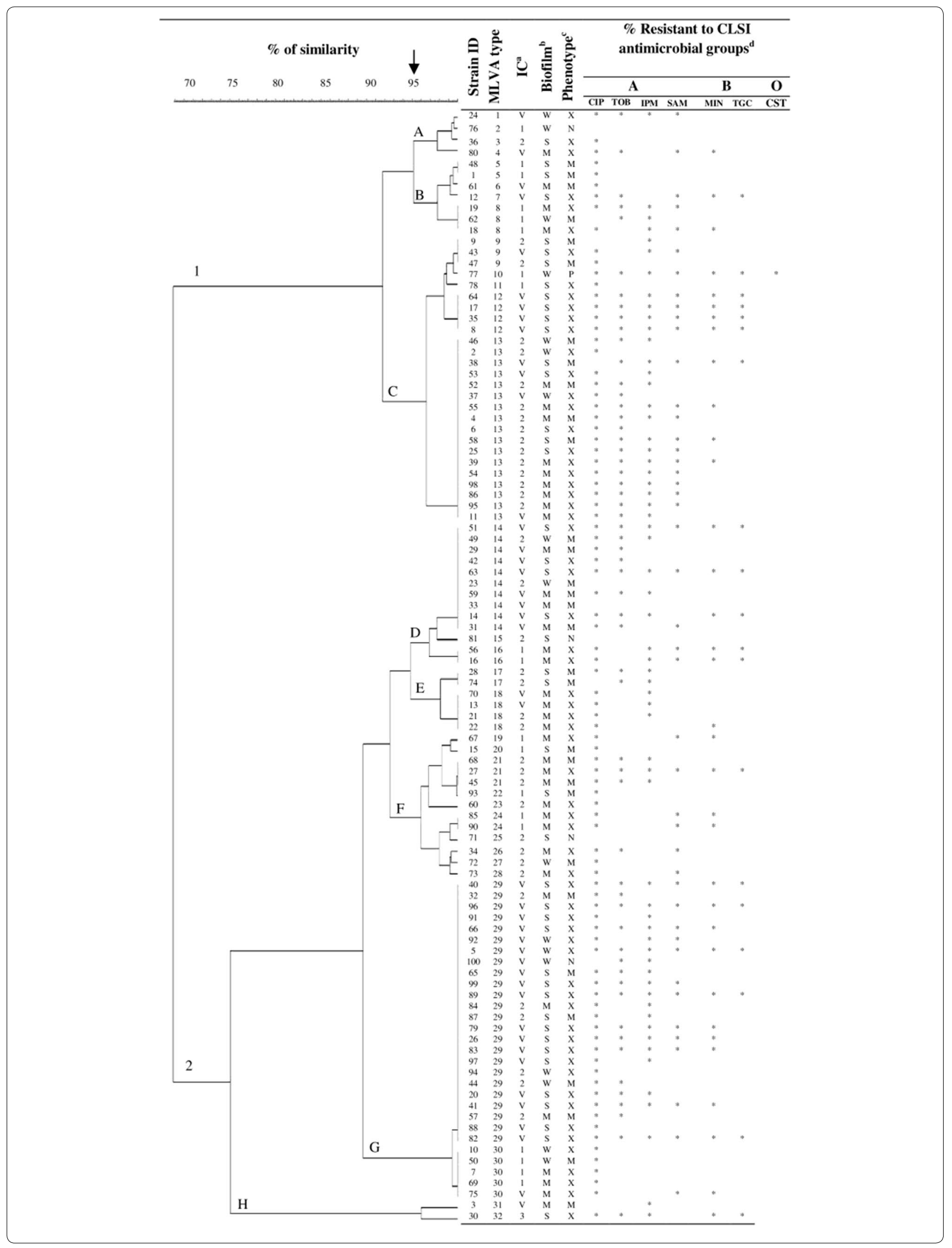


to form biofilm as a virulence determinant, as well as the IC type of each isolate (Fig. 2a, b). Our analysis focused on comparing the biofilm formation index of each isolate with the isolate MDR and XDR profile, since $95 \%$ of all isolates had either an MDR or XDR profile. Figure 2a shows that the majority of XDR isolates were IC variants, whereas MDR isolates were predominantly IC-II type. The frequency of IC-II isolates with MDR profile (55\%) was almost two folds higher than that of those with XDR profiles $(28 \% ; P=0.031)$. In contrast, the frequency of IC-V isolates with MDR profiles was nearly half the frequency of those with XDR profiles $(23 \%$ vs. $53 \% ; P=0.021)$. The frequencies of MDR vs. XDR profiles among IC-I and IC-III isolates was similar (Fig. 2a).

Comparison of the antimicrobial resistance profile of the isolates' with their biofilm strength index revealed that $77 \%$ of the $\mathrm{W}$ - and M-biofilm forming isolates had either an MDR, or XDR profile. While, the S-biofilm formers comprised only $23 \%$ of MDR isolates, the single PDR isolate was a W-biofilm former. All MT12 members $(n=4)$ were IC-V type and S-biofilm formers that showed an XDR antimicrobial susceptibility profile. Among XDR isolates, the frequency of S- and
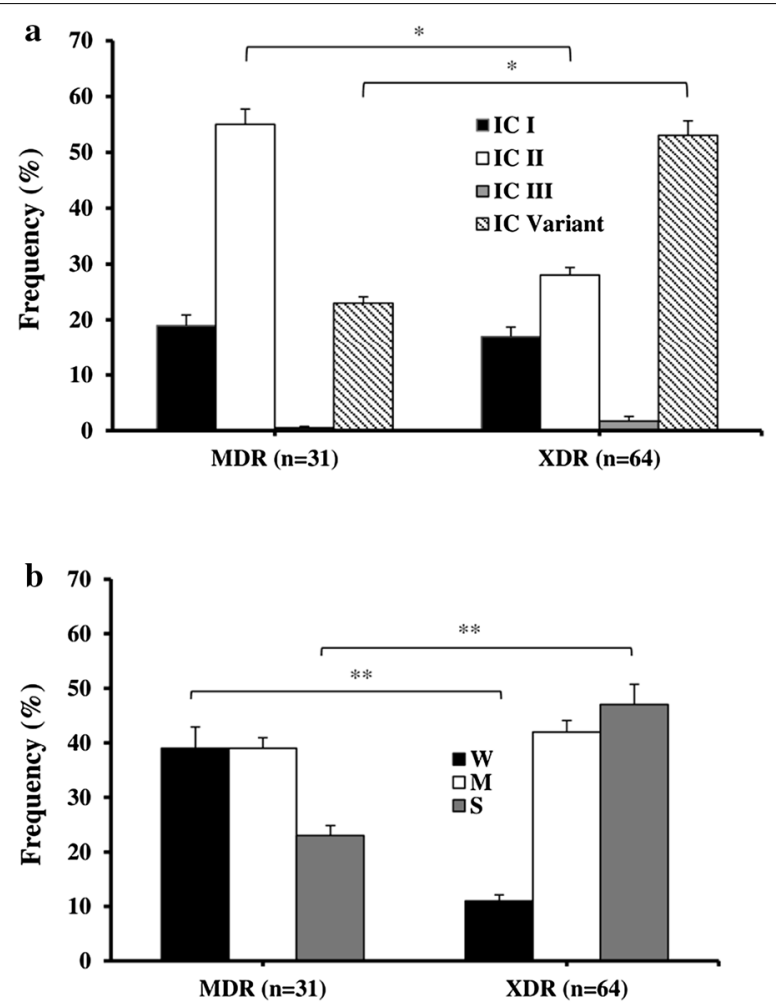

Fig. 2 Comparison of MDR and XDR isolates' frequency according to their IC type $\mathbf{a}$; and, the frequency of MDR and XDR isolates with weak $(W)$, moderate $(M)$, and strong $(S)$ biofilm formation $\mathbf{b}$. Bars indicate Mean $+S E M$; ${ }^{*} P<0.05 ;{ }^{* *} P<0.01$, between the indicated pairs
M-biofilm formers were 48 and 43\%, respectively; while among the MDR isolates, W- and M-biofilm formers were isolated at an identical $39 \%$ rate (Fig. 2b). In contrast, the frequency of W-biofilm formers among MDR isolates was $39 \%$, whereas only $12 \%$ of XDR isolates were W-biofilm formers $(P=0.009)$. Similarly, the frequency of S-biofilm forming isolates with the XDR, or MDR antimicrobial susceptibility profile was 47 and $23 \%$, respectively $(P=0.004)$. Finally, all four non-MDR isolates were either W- or S-biofilm formers (50\% frequency of isolation); i.e. they included no M-biofilm formers.

\section{Association of IC type with biofilm formation}

Additionally, we assessed whether the strength of biofilm formation was associated with the IC type of $A$. baumannii isolates. Figure 3 shows the frequency of W-, M- and S-biofilm formers among IC-I, IC-II, and IC-V isolates, which comprised $99 \%$ of all isolates. Among IC-V isolates, the frequency of S-biofilm formers (63\%) was six folds higher than the W-biofilm formers (12\%; $P=0.001)$; while, among IC-I and IC-II isolates, the frequencies of $\mathrm{W}-, \mathrm{M}-$-, and S-biofilm formers were comparable, showing only $4-7 \%$ variation: i.e. $26 \%$ vs. $19 \%$ for W-biofilm formers $(P=0.382)$, and $26 \%$ vs. $30 \%$ for $\mathrm{S}$-biofilm formers $(P=0.521)$, and finally $47 \%$ vs. $51 \%$ for M-biofilm formers $(P=0.437)$, in that order. As shown in Fig. 3, M-biofilm-forming isolates were almost twice as often seen among the IC-I and IC-II isolates compared to the IC-V isolates [47\% vs. $26 \%$ for IC-I $(P=0.036)$, and $51 \%$ vs. $26 \%$ for IC-II $(P=0.028)$, respectively.

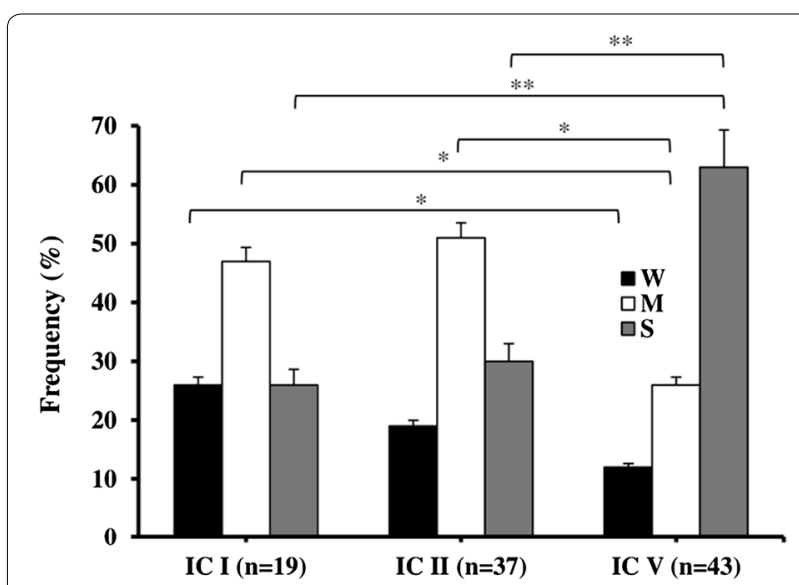

Fig. 3 Frequency comparison of weak (W), moderate (M), and strong (S) biofilm forming A. baumannii isolates according to their IC lineage. Analysis included semi-quantitative measurements of biofilm formation strength (see "Methods" section). Bars indicate Mean + SEM; ${ }^{*} \mathrm{P}<0.05$; ${ }^{*} \mathrm{P}<0.01$ 
Quantification of CFU concentration in wound bed tissues There was no significant difference between the log CFU concentrations in the wound sites infected with untreated Cst-R-AB isolate $(7.78 \pm 6.0)$, versus the log CFU concentrations $(6.99 \pm 5.82 ; \mathrm{P}>0.05)$ in wound beds that were infected with Cst-R-AB, which was treated with sub-MIC colistin. Thus, Cst-R-AB isolate concentrations (i.e. growth) were unaffected by the Cst-treatment of wound bed infection site.

\section{Virulence genes detection and expression in vitro and in vivo}

Virulence factor genes $d n a K, \operatorname{rec} A, \operatorname{lps} B$ and $b l s A$, were detected in the Cst-R-AB strain by PCR. In order to examine whether the expression of virulence genes dnaK, $b l s A, \operatorname{rec} A$, and $\operatorname{lps} B$ is associated with Cst resistance among isolates, we compared the relative gene-specific mRNA quantities of Cst-R-AB isolates under in vivo, vs. in vitro conditions (Fig. 4). The final data analysis included five Cst-R-AB isolates; including a single Cst$\mathrm{R}-\mathrm{AB}$ isolate from the present study (Additional file 1: Figure S1), and four additional Cst-R-AB isolates from previous studies.

Overall, as shown in Fig. 4, the expression levels of $d n a K$, recA, and $l p s B$ genes in Cst- $\mathrm{R}-\mathrm{AB}$ isolates were markedly different between in vivo vs. in vitro conditions. The relative change over basal expression levels of the four virulence genes, in vivo vs. in vitro, among Cst$\mathrm{R}-\mathrm{AB}$ isolates was reported as the Mean $\pm \mathrm{SD}$ (Fig. 4).

Among the Cst-R-AB isolates, the in vivo expression of $d n a K$ and lpsB was 23 and 14 folds higher than basal; respectively (Fig. 4); while $\operatorname{rec} A$ mRNA levels showed a slight 3 -fold increase $(P<0.05)$. Conversely, Cst-R-AB in vitro cultures revealed no significant increase in the expression of $\operatorname{dnaK}, b l s A$, and $\operatorname{lps} B$, with only a modest

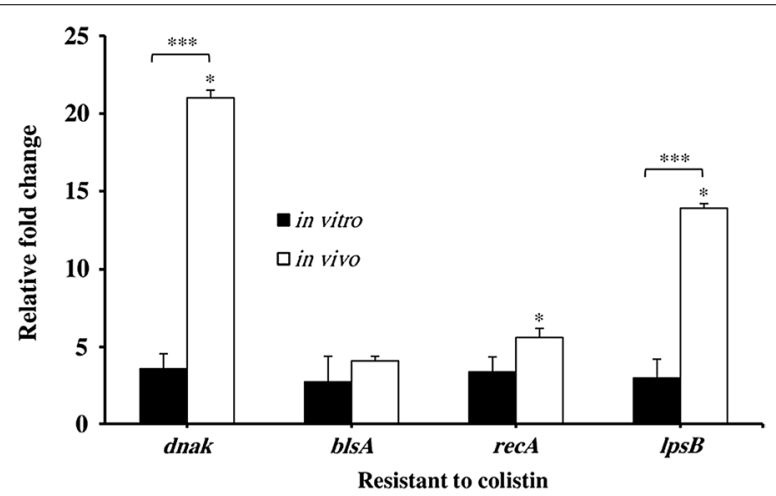

Fig. 4 Comparison of relative change in the mRNA levels of virulence determinant genes $d n a K, b / s A$, rec $A$, and $I p s B$ in cultures of five Cst-R-AB isolates, under in vitro and in vivo conditions. Bar indicates mean $\pm S D$; five mice in each group. ${ }^{\#} P<0.01$; ${ }^{*} P<0.05$; ${ }^{* * *} \mathrm{P}<0.001$ 4-fold rise in $\operatorname{rec} A$ expression. Interestingly, under either condition, $b l s A$ gene mRNA levels of the Cst-R-AB isolates was up-regulated by 3-4-fold.

\section{Discussion}

The emergence of multidrug-resistant $A$. baumannii (MDR-AB), as a major nosocomial pathogen among patients with burn wound infections, has drawn clinical attention to an old antimicrobial agent that is active against MDR-AB, namely colistin (Cst) [43, 44]. Unfortunately, reports from several regions of the world, including Iran, indicate that the widespread use of Cst, as a last resort to control MDR-AB infections, has led to a worrisome growing trend of Cst-resistance $\left(\mathrm{Cst}^{\mathrm{R}}\right)$ among MDR-AB strains $[8,45,46]$. Recurrently, the eradication of MDR-AB burn wound infections prove very challenging because, not only do clinical strains often develop antimicrobial resistance, but these strains also produce virulence factors (e.g. biofilm) that transform these MDR$\mathrm{AB}$ into even more formidable wound pathogens $[10,12]$.

While, some studies have associated the presence of specific virulence genes with an increase in the pathogenic potential of clinical isolates $[47,48]$, the association between the virulence gene expression of A. baumannii and the susceptibility to specific antimicrobial agents has not been assessed. In fact, despite several reports regarding the increase in the prevalence of Cst-resistance among MDR-AB isolates worldwide [8-14, 45], few studies have focused on the possible link between $\mathrm{Cst}^{\mathrm{R}}$, and the expression of virulence genes that can potentially affect therapy outcome. We have used a murine burn infection model to demonstrate that mRNA levels of virulence genes $d n a K, b l s A$, rec $A$, and $l p s B$ vary in Cst-R-AB isolates depending on whether they are cultured in vitro or in vivo. Moreover, to examine the genotypic diversity among $A$. baumannii isolates, we have characterized their MLVA genotypes, IC lineage. We further evaluated any associations between the isolates' IC lineage, susceptibility patterns, and their biofilm formation profiles.

Here, we also present evidence suggesting that the XDR or MDR profile of $A$. baumannii is associated with the biofilm strength. As shown in Fig. 2b, the frequency of W-biofilm formers among MDR isolates was about three times higher than among XDR isolates, which suggests that the strength of biofilm formation is related to the extended spectrum of antimicrobial resistance among isolates. Conversely, W- and M-biofilm formers were equally common (39\%) among MDR isolates. That the XDR isolates had more $\mathrm{M}$ - and S-biofilm formers than MDR isolates suggests that the antimicrobial susceptibility profile is associated with biofilm strength among these isolates. However, since the rates of M-biofilm formation were similar among XDR and MDR isolates, the 
M-biofilm formation phenotype does not seem to follow this association pattern.

Interestingly, the frequency of S- and M-biofilm formers among XDR isolates were similar ( $48 \%$ vs. $43 \%$, respectively); whereas among the MDR isolates, the Wand M-biofilm formers were isolated at an identical 39\% rate (Fig. 2b). Conversely, the frequency of W-biofilm formers among MDR isolates were about three times higher than XDR isolates $(P=0.009)$, which suggests that strength of biofilm formation is associated with the isolate antimicrobial profile. Likewise, isolates with XDR antimicrobial susceptibility profiles were more than twice as likely to be S-biofilm formers, as compared to the frequency of $\mathrm{S}$-formers among MDR isolates $(P=0.004)$. This suggests that stronger biofilm formation may be associated with broader antimicrobial resistance. The remarkable finding that all MT12 members were S-biofilm formers, and shared the same IC-V type and antimicrobial susceptibility profile is suggestive of a common source of isolation.

Interestingly, while IC- $\mathrm{V}$ variants had the highest frequency of S-biofilm formers, they included lowest frequency of W-biofilm formers. In addition, our data revealed that most of S-biofilm forming $\mathrm{IC}-\mathrm{V}$ isolates also had XDR profiles, which is contrary to the reports that indicate biofilm formation is a common attribute of clinical A. baumannii isolates, regardless of their IC minor or major clonal variation [49-52]. This apparent discrepancy might be due to the unique features of our local IC-V isolates that have neither been isolated, nor characterized elsewhere. Consequently, the conclusion of studies that have reported no correlation between $A$. baumannii biofilm formation and IC lineage may be limited to the IC-I, -II, and -III categories, which apparently do not encompass a global representation of all A. baumannii isolates.

Here, we present evidence suggesting that the development of $\mathrm{Cst}^{\mathrm{R}}$ may also lead to changes in A. baumannii virulence determinant genes expression, which in turn might enhance the ability to establish, and persist in burn wound infections. In order to further strengthen the scope of our initial findings, which were based on a single Cst- $\mathrm{R}-\mathrm{AB}$ isolate, we expanded our in vivo experimental data by using four additional Cst-R-AB isolates in a similar murine model (Fig. 4). Since $\mathrm{Cst}^{\mathrm{R}}$ has been shown to cause increased outer membrane permeability, higher division rate, and structural changes in the cell wall of A. baumannii isolates, it could be proposed that during prolonged infections, persistent $\mathrm{Cst}^{\mathrm{R}}$ can lead to reduced fitness, and lower A. baumannii virulence over time. However, our findings suggest that the opposite may occur, where enhanced $A$. baumannii virulence can be of greater benefit to some highly resistant local variants of MDR-AB. While the present study focused on the expression profile of four virulence genes among five Cst- $\mathrm{R}-\mathrm{AB}$ isolates, the variation in the levels of virulence gene mRNA in vitro vs. in vivo conditions among Cst-R$\mathrm{AB}$ isolates could be due to a myriad of additional factors, such as differences between the cellular environment of the wound site as compared to the cell-free culture conditions. Moreover, the role of tissue specific factors that dictate gene regulation, non-specific immune responses, remains to be investigated.

To our knowledge, this is the first report that attempts to establish an association between $\mathrm{Cst}^{\mathrm{R}}$ among $\mathrm{A}$. baumannii isolates and changes in the virulence genes expression. Our findings show the merits of a comprehensive investigation using a larger number of Cst-R-AB isolates from various regions of the world. Future detailed studies will shed light on the causal relationship between expression of a wider array of $A$. baumannii virulence genes, and genes that confer resistance to colistin, as well as other antimicrobial agents.

Finally, our findings highlight the intricate relationship between resistance to colistin, and the expression of $A$. baumannii virulence genes. It is commonly supposed that an increase in antimicrobial resistance leads, directly or indirectly, to reduced virulence of A. baumannii isolates. However, our data suggest that the opposite may occur, and it provides evidence that thorough investigations are warranted to directly measure the correlation between $\mathrm{Cst}^{\mathrm{R}}$, reduced virulence, and "fitness" of A. baumannii isolates. Virulence gene expression variations among Cst-R-AB isolates might gain further clinical importance in health care settings, where isolates often transfer from patient to patient. Moreover, that the virulence of Cst-R-AB isolates may depend on their in vitro or in vivo conditions can help explain, at least in part, commonly encountered discrepancies between in vivo and in vitro laboratory test results. For instance, the recurrent failure of therapeutic antimicrobial agents in eradicating $A$. baumannii in patients' burn wounds, despite the in vitro susceptibility of isolated pathogen to the same agents.

\section{Conclusions}

This study concludes that the development of resistance to colistin among $A$. baumannii may be associated with virulence gene expression, which fluctuates under in vivo and in vitro conditions. We also provide evidence to speculate that enhanced virulence may be of greater benefit to highly-resistant variants of MDR-AB, and may give rise to formidable pathogens that increase morbidity among burn patients, and threaten the public health systems worldwide. Our findings warrant detailed examination of the interactions between virulence and antimicrobial 
resistance toward efforts to control the spread of multidrug-resistant $A$. baumannii (MDR-AB) isolates, and also to reduce disease severity in burn patients with MDR-AB infection.

\section{Additional file}

Additional file 1: Figure S1. Comparison of relative change in the mRNA levels of virulence determinant genes $d n a K, b / s A$, recA, and Ips $B$ in cultures of a single Cst-R A. baumannii isolate, under in vitro and in vivo conditions. Bar indicates Mean + SD; five mice in each group. ${ }^{\#}<0.01$; ${ }^{*} P<0.05 ;{ }^{* *} \mathrm{P}<0.001$.

\section{Abbreviations}

AB: Acinetobacter baumannii or A. baumannii; MDR-AB: multidrug-resistant $A$. baumannii; Cst-R-AB: colistin-resistant A. baumannii; recA: gene encoding the RecA protein; Ips $B$ : gene encoding the lipopolysaccharide core biosynthesis mannosyltransferase protein $L p s B$; dnaK: gene encoding the chaperone protein DnaK; blsA: gene encoding the BlsA (blue-light-sensing) photoreceptor protein; MDR: multi-drug resistant; XDR: extensive-drug resistant; GmbH: Gesellschaft mit beschränkter Haftung, which means "company with limited liability"; Cst ${ }^{R}$ : colistin resistance; TUMS: Tehran University of Medical Sciences; gyrB: gene encoding the GyrB subunit of DNA gyrase protein; PCR: polymerase chain reaction; MHB: Mueller-Hinton Broth; DAD: disk agar diffusion; CLSI: Clinical and Laboratory Standards Institute; MIC: minimum inhibitory concentration; EUCAST: European Committee on Antimicrobial Susceptibility Testing; LPS: lipopolysaccharide; C-Burn: Mock-infected burned mice that serve as controls; ompA: gene encoding outer membrane protein $A ; b / a_{O X A-5}$ : the gene encoding the carbapenemase OXA-51; ompA: outer membrane protein $A$; csuE: chaperone-subunit usher E; MLVA: multi-loci variable-number tandem repeat analysis; DNA: deoxyribonucleic acid; ng: nanogram; $\mu \mathrm{L}$ : microliter; mM: millimolar; $\mathrm{MgCl}_{2}$ : magnesium chloride; U: units; Taq: Thermus aquaticus; $\mu \mathrm{M}$ : micromolar; MIQE: minimum information for publication of quantitative real-time PCR experiments; dNTP: deoxyribonucleotide triphosphate; et al.: Et alii meaning "and other people"; s: second(s); $\mathrm{cm}$ : centimeter(s); \%: percent; $h$ : hour(s); V: volt(s); X: times; TBE: tris-borate-EDTA; bp: base pair(s); VNTRs: variable number tandem repeats; L-VNTRs: large variable number tandem repeats: S-VNTRs: small variable number tandem repeats; $\mu \mathrm{g}$ : microgram(s); v.: version; BHI: brain-heart-infusion; LB: Luria-Bertani; CFU: colony forming unit(s); $\mathrm{dH}_{2} \mathrm{O}$ : distilled water; RT: room temperature; A: optical absorbance; Nm: nanometer(s); PDR: pan-drug resistant; ATCC: American Type Culture Collection; NC: negative control; N: negative isolate; M: moderately positive isolate; $S$ : strongly positive isolate; $A_{i}$ initial absorbance; $A_{N}$ : absorbance of negative control; ${ }^{\circledR}$ : registered trademark; wk: week; g: gram; No.: number; IC: international clone; i.p.: intraperitoneal; mm: millimeter; kg: kilogram; RNA: ribonucleic acid; cDNA: complementary DNA; PBS: phosphate-buffered saline; $\mathrm{N}_{2}$ : nitrogen; S: svedberg unit(s) (e.g. 16S); rRNA: ribosomal ribonucleic acid; mRNA: mitochondrial ribonucleic acid; USA: United States of America; RT-PCR: reverse transcription PCR; qRT-PCR: quantitative real-time RT-PCR; DNase I: deoxyribonuclease $\mathrm{I}^{\circ} \mathrm{C}$ : degrees centigrade; pmol: picomolar; ng: nanogram(s); min: minute(s); $\mathrm{ddH}_{2} \mathrm{O}$ : double distilled water; REST: Relative Expression Software Tool; >: greater than; $n$-fold difference: Relative fold difference as compared to the basal gene expression; MT: MLVA type(s); W-biofilm formers: weak biofilm formers; M-biofilm formers: moderate biofilm formers; S-biofilm formers: strong biofilm formers; Suppl.: supplement; SD: standard deviation.

\section{Authors' contributions}

$\mathrm{FBH}$ and $\mathrm{AB}$ analyzed and processed the data and drafted the manuscript. ZF and MM did the PCR and MLVA assays. MM did the specimen cultures, animal studies and biofilm assays. MP performed the antimicrobial assays. RR helped with the RNA extraction and virulence gene expression assays, BP helped with clinical coordination and obtaining the patient specimens from the hospitals. All authors read and approved of the final manuscript.

\section{Author details}

1 Department of Microbiology, School of Medicine, Tehran University of Medical Sciences, 100 Poursina Ave., 100 Keshavarz Blvd, Tehran 14167-53955, Iran.
${ }^{2}$ Legal Medicine Research Center, Legal Medicine Organization, Tehran, Iran. ${ }^{3}$ Innovative Research Center, Islamic Azad University, Mashhad Branch, Mashhad, Iran. ${ }^{4}$ Pediatrics Infectious Disease Research Center, Tehran University of Medical Sciences, Tehran, Iran. ${ }^{5}$ Dental Research Center, Dentistry Research Institute, Tehran University of Medical Sciences, Tehran, Iran. ${ }^{6}$ Laser Research Center, Dentistry Research Institute, Tehran University of Medical Sciences, Tehran, Iran.

\section{Acknowledgements}

We would like to thank all the medical staff, who assisted with collection of the specimens used in this study. We would also like to acknowledge Ms. Jocelyn C. Gile for her invaluable editorial assistance.

\section{Competing interests}

The authors declare that they have no competing interests.

\section{Document availability}

All documents and additional data are available from the corresponding author upon reasonable request.

\section{Ethics approval and consent to participate}

This study was approved by Tehran University of Medical Sciences (TUMS) Research Ethics Committee for Human Research (Application No. TRECHR-89. 01-30-10430). Written informed consent forms were obtained from either the patients, or their authorized representatives. All animal experiments were carried out in accordance to the protocols approved by the Animal Ethics Committee of Tehran University of Medical Sciences.

\section{Funding}

This research was supported by Tehran University of Medical Sciences \& Health Services Grant No. 89. 01-30-10430.

\section{Publisher's Note}

Springer Nature remains neutral with regard to jurisdictional claims in published maps and institutional affiliations.

Received: 12 February 2018 Accepted: 19 May 2018

Published online: 01 June 2018

\section{References}

1. Girerd-Genessay I, Bénet T, Vanhems P. Multidrug-resistant bacterial outbreaks in burn units: a synthesis of the literature according to the ORION statement. J Burn Care Res. 2016;37(3):172-80.

2. Leseva M, Arguirova M, Nashev D, Zamfirova E, Hadzhyiski O. Nosocomial infections in burn patients: etiology, antimicrobial resistance, means to control. Ann Burns Fire Disasters. 2013:26(1):5-11.

3. Golberg A, Broelsch GF, Vecchio D, et al. Eradication of multidrug-resistant A. baumannii in burn wounds by antiseptic pulsed electric field. Technology (Singap World Sci). 2014;2(2):153-60.

4. Atilla A, Tomak L, Katrancı AO, Ceylan A, Kılıç SS. Mortality risk factors in burn care units considering the clinical significance of acinetobacter infections. Ulus Travma Acil Cerrahi Derg. 2015;21(1):34-8.

5. Mortensen BL, Skaar EP. Host-microbe interactions that shape the pathogenesis of Acinetobacter baumannii infection. Cell Microbiol. 2012;14(9):1336-44.

6. Longo F, Vuotto C, Donelli G. Biofilm formation in Acinetobacter baumannii. New Microbiol. 2014;37(2):119-27.

7. Weber BS, Harding CM, Feldman MF. Pathogenic acinetobacter: from the cell surface to infinity and beyond. J Bacteriol. 2015;198(6):880-7.

8. Bahador A, Taheri M, Pourakbari B, et al. Emergence of rifampicin, tigecycline, and colistin-resistant Acinetobacter baumannii in Iran; spreading of MDR strains of novel international clone variants. Microb Drug Resist. 2013;19(5):397-406.

9. Moradi J, Hashemi FB, Bahador A. Antibiotic resistance of Acinetobacter baumannii in Iran: a systemic review of the published literature. Osong Public Health Res Perspect. 2015;6(2):79-86. 
10. Doi Y, Murray GL, Peleg AY. Acinetobacter baumannii: evolution of antimicrobial resistance-treatment options. Semin Respir Crit Care Med. 2015;36(1):85-98

11. Inchai J, Liwsrisakun C, Theerakittikul T, Chaiwarith R, Khositsakulchai W, Pothirat C. Risk factors of multidrug-resistant, extensively drug-resistant and pandrug-resistant Acinetobacter baumannii ventilator-associated pneumonia in a Medical Intensive Care Unit of University Hospital in Thailand. J Infect Chemother. 2015;21(8):570-4.

12. Potron A, Poirel L, Nordmann P. Emerging broad-spectrum resistance in Pseudomonas aeruginosa and Acinetobacter baumannii: mechanisms and epidemiology. Int J Antimicrob Agents. 2015;45(6):568-85.

13. Gupta M, Lakhina K, Kamath A, et al. Colistin-resistant Acinetobacter baumannii ventilator-associated pneumonia in a tertiary care hospital: an evolving threat. J Hosp Infect. 2016;94(1):72-3

14. Liu H, Wu YQ, Chen LP, et al. Biofilm-related genes: analyses in multiantibiotic resistant Acinetobacter Baumannii isolates from Mainland China. Med Sci Monit. 2016;28(22):1801-7.

15. Zarrilli R. Acinetobacter baumannii virulence determinants involved in biofilm growth and adherence to host epithelial cells. Virulence. 2016;7(4):367-8.

16. Lee J, Page R, García-Contreras R, et al. Structure and function of the Escherichia coli protein YmgB: a protein critical for biofilm formation and acid-resistance. J Mol Biol. 2007;373(1):11-26.

17. Wroblewska MM, Sawicka-Grzelak A, Marchel H, Luczak M, Sivan A. Biofilm production by clinical strains of Acinetobacter baumannii isolated from patients hospitalized in two tertiary care hospitals. FEMS Immunol Med Microbiol. 2008;53(1):140-4.

18. Fiester SE, Actis LA. Stress responses in the opportunistic pathogen Acinetobacter baumannii. Future Microbiol. 2013;8(3):353-65.

19. Schröder W, Goerke C, Wolz C. Opposing effects of aminocoumarins and fluoroquinolones on the SOS response and adaptability in Staphylococcus aureus. J Antimicrob Chemother. 2013;68(3):529-38.

20. Aranda J, Bardina C, Beceiro A, et al. Acinetobacter baumannii RecA protein in repair of DNA damage, antimicrobial resistance, general stress response, and virulence. J Bacteriol. 2011;193(15):3740-7.

21. Beceiro A, Tomás M, Bou G. Antimicrobial resistance and virulence: a successful or deleterious association in the bacterial world? Clin Microbiol Rev. 2013;26(2):185-230.

22. Luke NR, Sauberan SL, Russo TA, et al. Identification and characterization of a glycosyltransferase involved in Acinetobacter baumannii lipopolysaccharide core biosynthesis. Infect Immun. 2010;78(5):2017-23.

23. McConnell MJ, Actis L, Pachón J. Acinetobacter baumannii: human infections, factors contributing to pathogenesis and animal models. FEMS Microbiol Rev. 2013;37(2):130-55.

24. Lean SS, Yeo CC, Suhaili Z, Thong KL. Comparative genomics of Two ST 195 carbapenem-resistant Acinetobacter baumannii with different susceptibility to polymyxin revealed underlying resistance mechanism. Front Microbiol. 2016;6:1445. https://doi.org/10.3389/fmicb.2015.01445.

25. Mussi MA, Gaddy JA, Cabruja M, et al. The opportunistic human pathogen Acinetobacter baumannii senses and responds to light. J Bacteriol. 2010;192(24):6336-45.

26. Golic A, Vaneechoutte M, Nemec A, Viale AM, Actis LA, Mussi MA. Staring at the cold sun: blue light regulation is distributed within the genus Acinetobacter. PLoS ONE. 2013;8(1):e55059. https://doi org/10.1371/journal.pone.0055059.

27. Pournaras S, Poulou A, Dafopoulou K, et al. Growth retardation, reduced invasiveness, and impaired colistin-mediated cell death associated with colistin resistance development in Acinetobacter baumannii. Antimicrob Agents Chemother. 2014;58(2):828-32.

28. Dafopoulou K, Xavier BB, Hotterbeekx A, et al. Colistin-resistant Acinetobacter baumannii clinical strains with deficient biofilm formation. Antimicrob Agents Chemother. 2015;60(3):1892-5.

29. Higgins PG, Schneiders T, Hamprecht A, Seifert H. In vivo selection of a missense mutation in adeR and conversion of the novel blaOXA-164 gene into blaOXA-58 in carbapenem-resistant Acinetobacter baumannii isolates from a hospitalized patient. Antimicrob Agents Chemother. 2010;54(12):5021-7.

30. Clinical and Laboratory Standards Institute. Performance standards for antimicrobial susceptibility testing: twenty fourth informational supplement M100-S28. Wayne: CLSI; 2018.
31. The European Committee on Antimicrobial Susceptibility Testing. Breakpoint tables for interpretation of MICs and zone diameters Version 5.0. 2015. http://www.eucastorg/clinical_breakpoints. Accessed 01 Jan 2015.

32. Magiorakos AP, Srinivasan A, Carey RB, et al. Multidrug-resistant, extensively drug-resistant and pandrug-resistant bacteria: an international expert proposal for interim standard definitions for acquired resistance. Clin Microbiol Infect. 2012;18(3):268-81.

33. Turton JF, Gabriel SN, Valderrey C, Kaufmann ME, Pitt TL. Use of sequence-based typing and multiplex PCR to identify clonal lineages of outbreak strains of Acinetobacter baumannii. Clin Microbiol Infect. 2007;13(8):807-15

34. Pourcel C, Minandri F, Hauck Y, et al. Identification of variable-number tandem-repeat (VNTR) sequences in Acinetobacter baumannii and interlaboratory validation of an optimized multiple-locus VNTR analysis typing scheme. J Clin Microbiol. 2011;49(2):539-48.

35. Nhu NT, Lan NP, Campbell Jl, et al. Emergence of carbapenem-resistant Acinetobacter baumannii as the major cause of ventilator-associated pneumonia in intensive care unit patients at an infectious disease hospital in southern Vietnam. J Med Microbiol. 2014;63(Pt10):1386-94.

36. Zhang $D, X i a ~ J, X u Y$, et al. Biological features of biofilm-forming ability of Acinetobacter baumannii strains derived from 121 elderly patients with hospital-acquired pneumonia. Clin Exp Med. 2016;16(1):73-80.

37. Bahador A, Raoo An R, Farshadzadeh Z, et al. The prevalence of IS Aba 1 and IS Aba 4 in Acinetobacter baumannii species of different international clone lineages among patients with burning in Tehran, Iran. Jundishapur J Microbiol. 2015;8(7):e17167. https://doi.org/10.5812/ jjm.17167v2.

38. Pachón-Ibáñez ME, Docobo-Pérez F, López-Rojas R, et al. Efficacy of rifampin and its combinations with imipenem, sulbactam, and colistin in experimental models of infection caused by imipenemresistant Acinetobacter baumannii. Antimicrob Agents Chemother. 2010:54(3):1165-72.

39. Dai T, Tegos GP, Lu Z, et al. Photodynamic therapy for Acinetobacter baumannii burn infections in mice. Antimicrob Agents Chemother. 2009;53(9):3929-34.

40. Ha U, Jin S. Expression of the soxR gene of Pseudomonas aeruginosa is inducible during infection of burn wounds in mice and is required to cause efficient bacteremia. Infect Immun. 1999;67(10):5324-31.

41. Thompson MG, Black CC, Pavlicek RL, et al. Validation of a novel murine wound model of Acinetobacter baumannii infection. Antimicrob Agents Chemother. 2014;58(3):1332-42.

42. Bustin SA, Beaulieu JF, Huggett J, Jaggi R, Kibenge FSB, Olsvik PA, Penning LC, Toegel S. MIQE précis: practical implementation of minimum standard guidelines for fluorescence-based quantitative realtime PCR experiments. BMC Mol Biol. 2010;11:74.

43. Saun TJ, Rogers AD, Leis JA, Cartotto R. The use of intravenous and inhaled colistin therapy during a burn center outbreak of multidrugresistant Acinetobacter baumannii. J Burn Care Res. 2017. https://doi. org/10.1097/BCR.

44. Fishbain J, Peleg AY. Treatment of acinetobacter infections. Clin Infect Dis. 2010;51(1):79-84.

45. Sepahvand S, Doudi M, Davarpanah MA, Bahador A, Ahmadi M. Analyzing pmrA and pmrB genes in Acinetobacter baumannii resistant to colistin in Shahid Rajai Shiraz, Iran Hospital by PCR: first report in Iran. Pak J Pharm Sci. 2016;29(4 Suppl):1401-6.

46. Garnacho-Montero J, Dimopoulos G, Poulakou G, et al. Task force on management and prevention of Acinetobacter baumannii infections in the ICU. Intensive Care Med. 2015;41(12):2057-75.

47. Eijkelkamp BA, Stroeher UH, Hassan KA, Paulsen IT, Brown MH. Comparative analysis of surface-exposed virulence factors of Acinetobacter baumannii. BMC Genomics. 2014;25(15):1020. https://doi. org/10.1186/1471-2164-15-1020.

48. Yoon EJ, Balloy V, Fiette L, Chignard M, Courvalin P, Grillot-Courvalin C. Contribution of the Ade resistance-nodulation-cell division-type efflux pumps to fitness and pathogenesis of Acinetobacter baumannii. MBio. 2016;7(3):pii: e00697-716. https://doi.org/10.1128/mbio.00697-16.

49. Lee NY, Chang TC, Wu CJ, et al. Clinical manifestations, antimicrobial therapy, and prognostic factors of monomicrobial Acinetobacter baumannii complex bacteremia. J Infect. 2010;61(3):219-27. 
50. Azim A, Dwivedi M, Rao PB, et al. Epidemiology of bacterial colonization at intensive care unit admission with emphasis on extended-spectrum beta-lactamase- and metallo-beta-lactamase-producing Gramnegative bacteria-an Indian experience. J Med Microbiol. 2010;59(Pt 8):955-60.

51. Kang YS, Park W. Contribution of quorum-sensing system to hexadecane degradation and biofilm formation in Acinetobacter sp. strain DR1. J Appl Microbiol. 2010;109(5):1650-9.
52. Sanchez CJ, Kumar N, Lizcano A, et al. Streptococcus pneumoniae in biofilms are unable to cause invasive disease due to altered virulence determinant production. PLoS ONE. 2011;6(12):e28738. https://doi. org/10.1371/journal.pone.0028738. 